\title{
Significant response to ramucirumab monotherapy in chemotherapy-resistant recurrent alpha-fetoprotein-producing gastric cancer: A case report
}

\author{
YASUHIRO ARAKAWA ${ }^{1}$, MIHO TAMURA ${ }^{1}$, KEISUKE AIBA ${ }^{1}$, KAZUHIKO MORIKAWA ${ }^{2}$, \\ DAISUKE AIZAWA $^{3}$, MASAHIRO IKEGAMI ${ }^{3}$, MASAMI YUDA $^{4}$ and KATSUNORI NISHIKAWA ${ }^{4}$ \\ Departments of ${ }^{1}$ Oncology and Hematology, ${ }^{2}$ Radiology, ${ }^{3}$ Pathology and ${ }^{4}$ Surgery, \\ Jikei University School of Medicine, Tokyo 105-8471, Japan
}

Received April 19, 2016; Accepted March 9, 2017

DOI: $10.3892 / \mathrm{ol} .2017 .6514$

\begin{abstract}
Alpha-fetoprotein (AFP)-producing gastric cancer (AFPGC) is a relatively rare type of gastric cancer characterized by a high incidence of liver and lymph node metastases, and a poor prognosis. Few advanced AFPGC cases treated successfully with conventional chemotherapy have been reported thus far. Although the development of molecular-targeted therapy has improved the prognosis of various types of cancer, there are currently no tailored therapies for AFPGC. In the present report, the case of a chemotherapy-resistant recurrent AFPGC patient who exhibited a significant response to ramucirumab monotherapy is presented. Following six doses of ramucirumab, a metastatic lymph node displayed central necrosis, and the patient's serum AFP levels decreased from 12,800 to $225 \mathrm{ng} / \mathrm{ml}$. AFPGC is known to have increased vascular endothelial growth factor (VEGF) expression and rich neovascularization. Furthermore, in the present case, tumor cells were positive for VEGF. Ramucirumab is a monoclonal antibody for VEGF receptor-2 and the first anti-angiogenic drug approved for the treatment of advanced gastric cancer. However, the clinical efficacy of ramucirumab in patients with AFPGC has not been reported previously. The present report suggests that AFP production in gastric cancer can be a predictor for the response to anti-angiogenic drugs such as ramucirumab.
\end{abstract}

\section{Introduction}

Alpha-fetoprotein (AFP)-producing gastric cancer (AFPGC) is a relatively rare type of malignancy, which comprises $1-8 \%$

Correspondence to: Dr Yasuhiro Arakawa, Department of Oncology and Hematology, Jikei University School of Medicine, Nishi-Shimbashi 3-19-18, Minato-Ku, Tokyo 105-8471, Japan E-mail: yarakawa@jikei.ac.jp

Key words: alpha-fetoprotein-producing gastric cancer, chemotherapy-resistant gastric cancer, recurrent gastric cancer, ramucirumab monotherapy, anti-angiogenic therapy of all gastric cancers (1-3). AFPGC is characterized by a high incidence of metastases to the liver and lymph nodes, and a poor prognosis (1-3). Currently, there is no standard therapy for this challenging subtype of gastric cancer. Histopathologically, AFPGC is known to have high proliferative activity, reduced apoptotic rate and rich neovascularization, which influence the clinical aggressiveness of the disease $(3,4)$. High expression of vascular endothelial growth factor (VEGF) is considered one of the main reasons for the rich neovascularization in AFPGC (3-5).

Over the past few decades, molecular-targeted therapy has led to improved survival of patients with various types of cancer, including gastric cancer (6). A humanized anti-human epidermal growth factor receptor 2 (HER2) monoclonal antibody (namely trastuzumab) in combination with chemotherapy demonstrated a survival benefit for patients with advanced HER2-positive gastric cancer (7). A monoclonal antibody for VEGF receptor-2 (namely ramucirumab) also demonstrated survival benefit for patients with advanced gastric cancer following first-line chemotherapy $(8,9)$.

Ramucirumab binds to VEGF receptor-2 and inhibits VEGF-induced angiogenesis $(8,9)$. Ramucirumab is the first anti-angiogenic drug approved for the treatment of advanced gastric cancer; however, there is no validated predictor for selecting patients who will benefit from ramucirumab therapy to date $(8,9)$. Although AFPGC has rich neovascularization $(3,4)$, the clinical efficacy of ramucirumab in patients with AFPGC has not yet been reported. In the current case report, the case of a chemotherapy-resistant recurrent AFPGC patient who received ramucirumab monotherapy is presented.

\section{Case report}

A 56-year-old man with upper abdominal pain was referred to Jikei University Hospital (Tokyo, Japan) in January 2014. The patient had no significant family or medical history, except well-controlled hypertension. Gastrointestinal endoscopy revealed a 5-cm elevated tumor with a central depression (Borrmann type 3 ) on the posterior wall of the gastric body, which was histologically diagnosed as moderately differentiated tubular adenocarcinoma. HER2 
immunostaining was performed using the HercepTest kit (Dako; Agilent Technologies, Inc., Santa Clara, CA, USA) and scored as 1+, according to the manufacturer's instruction (10). Computed tomography (CT) revealed thickening of the gastric wall and enlargement of lymph nodes on the lesser curvature. There were no signs of liver metastases or liver cirrhosis. The results of a complete blood count and blood chemistry were as follows: Hemoglobin $14.4 \mathrm{~g} / \mathrm{dl}$ (normal range, 13.5-16.5 g/dl), white blood cell count $6,300 / \mu 1$ (normal, 3,300-8,600/ $\mu 1$ ), platelet count $227,000 / \mu 1$ (normal, 150,000-350,000/ $\mu 1$ ), aspartate aminotransferase $22 \mathrm{U} / 1$ (normal, 10-33 U/l), alanine aminotransferase $14 \mathrm{U} / 1$ (normal, 6-35 U/l), total protein $7.3 \mathrm{~g} / \mathrm{dl}$ (normal, 6.7-8.3 g/dl), albumin 4.2 g/dl (normal, 3.5-5.2 g/dl), blood urea nitrogen $11 \mathrm{mg} / \mathrm{dl}$ (normal, $8-20 \mathrm{mg} / \mathrm{dl}$ ), creatinine $0.55 \mathrm{mg} / \mathrm{dl}$ (normal, $0.50-1.10 \mathrm{mg} / \mathrm{dl}$ ), and C-reactive protein (CRP) $0.08 \mathrm{mg} / \mathrm{dl}$ (normal, $0-0.30 \mathrm{mg} / \mathrm{ml}$ ). Hepatitis $\mathrm{B}$ surface antigen and hepatitis $\mathrm{C}$ antibody were negative. The patient's serum AFP and carcinoembryonic antigen (CEA) levels were increased to $910 \mathrm{ng} / \mathrm{ml}$ (normal range, $0-10 \mathrm{ng} / \mathrm{ml}$ ) and $17.0 \mathrm{ng} / \mathrm{ml}$ (normal range, $0.0-5.8 \mathrm{ng} / \mathrm{ml}$ ), respectively.

The patient received two courses of neoadjuvant chemotherapy with S-1 (tegafur/gimeracil/oteracil potassium) plus cisplatin (11), which resulted in stable disease, and then underwent distal gastrectomy with D2 lymph node dissection (Fig. 1A). Histopathologically, the tumor was predominantly composed of well- to moderately-differentiated adenocarcinoma with papillary and tubular proliferation (Fig. 1B). The specimen also contained minor components of poorly differentiated adenocarcinoma with signet-ring cells. The typical histological feature of AFPGC is a liver-like structure called a 'hepatoid', but the specimen did not contain a hepatoid component (12). Immunohistological staining for AFP and VEGF was performed by the avidin-biotin complex (ABC) method. Following deparaffinization and rehydration, 4- $\mu \mathrm{m}$ thick sections were treated with $0.3 \% \mathrm{H}_{2} \mathrm{O}_{2}$ for 30 min and blocked with $1 \%$ bovine serum albumin in phosphate-buffered saline (PBS) for $30 \mathrm{~min}$. Then, sections were rinsed in PBS and treated with antibodies against AFP (dilution, 1:100; cat. no A008; Dako; Agilent Technologies, Inc.) or VEGF (dilution, 1:100; cat. no R11; Immuno-Biological Laboratories Co., Ltd., Gunma, Japan) for $1 \mathrm{~h}$ at room temperature in a humidified chamber, followed by incubation with biotinylated $\operatorname{IgG}$ (dilution, 1:100; Vector Laboratories, Burlingame, CA, USA) for $30 \mathrm{~min}$ at room temperature. The sections were developed using ABC-peroxidase and diaminobenzidine (both Vector Laboratories). AFP-positive foci were observed (Fig. 1C) and VEGF expression was positive in the cytoplasm of the tumor cells (Fig. 1D). The final stage was T3, N2, M0, stage IIIA, according to the 7th edition of the Union for the International Cancer Control classification (13), and a curative resection was confirmed. A month after the surgery, the patient's serum AFP and CEA levels were decreased to 15 and $3.8 \mathrm{ng} / \mathrm{ml}$, respectively. The patient was started on adjuvant therapy with S-1 (14) (Fig. 2).

Following a course of S-1 therapy ( 3 months afterthe surgery), $\mathrm{CT}$ examination revealed a $22-\mathrm{mm}$ para-aortic lymph node. The serum AFP levels were increased to $207 \mathrm{mg} / \mathrm{ml}$, and the patient was diagnosed as having a recurrence of AFPGC. The patient was then treated with five courses of weekly paclitaxel (14),
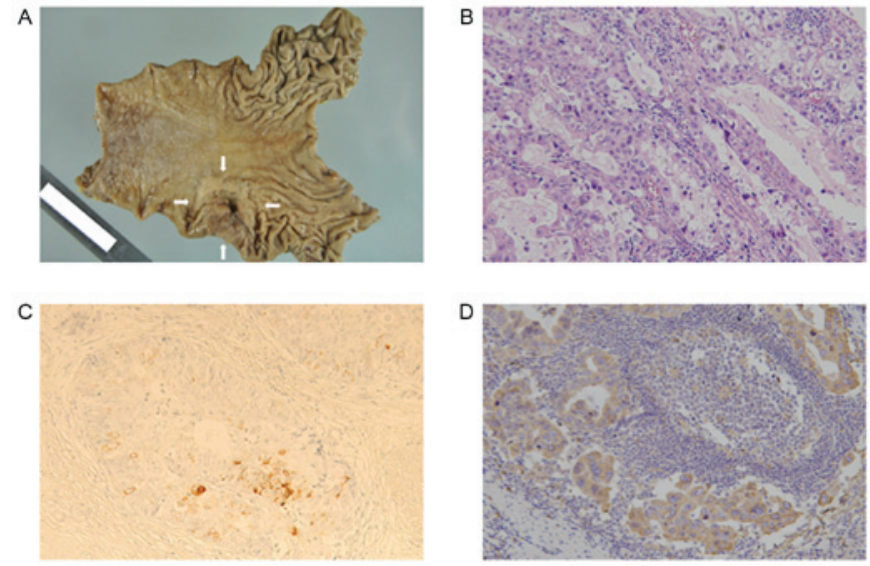

Figure 1. (A) Image of the resected specimen, showing a Borrmann type 3 tumor of $55 \times 40 \mathrm{~mm}$ in size located at the body of the stomach (as indicated by the arrows). (B) Histopathological examination revealed moderately differentiated adenocarcinoma components with papillary and tubular proliferation (hematoxylin and eosin staining; magnification, x100). (C) Immunohistological staining demonstrated alpha-fetoprotein-positive foci (magnification, x100). (D) Immunoreactivity for vascular endothelial growth factor was observed in the cytoplasm of cancer cells (magnification, $\mathrm{x} 100)$.
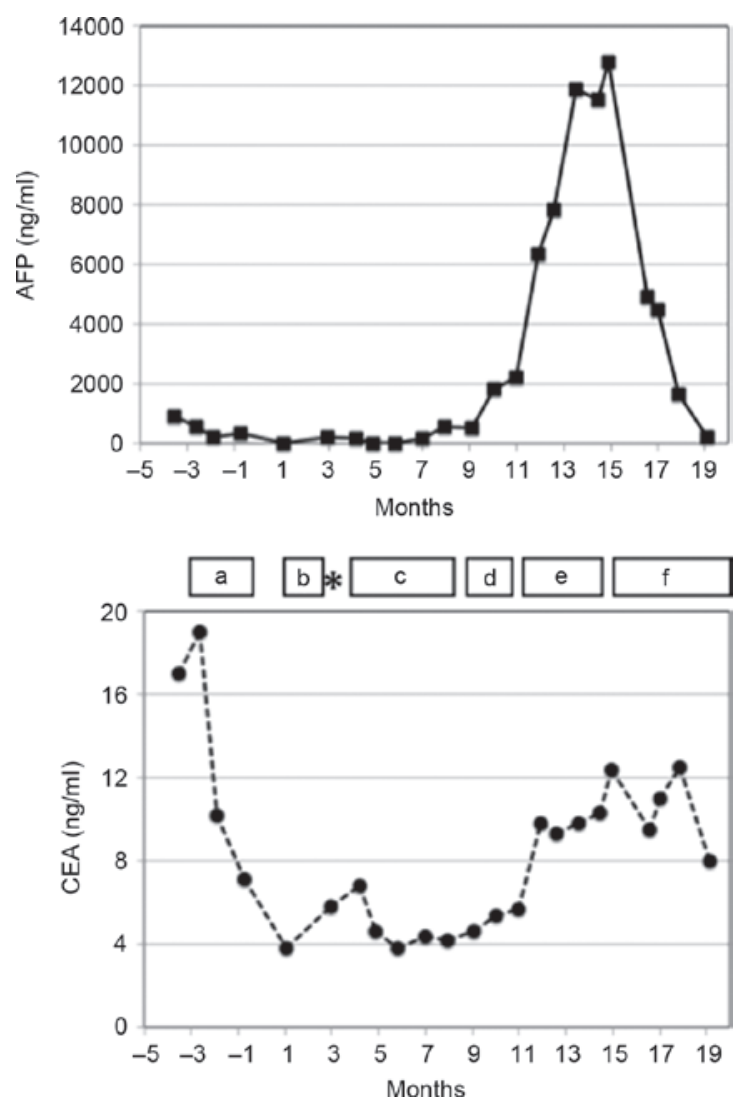

Figure 2. Graph depicting the patient's therapeutic course and serum tumor marker levels. The patient received the following treatments: a, S-1 (tegafur/gimeracil/oteracil potassium) plus cisplatin; b, S-1; c, paclitaxel; d, irinotecan; e, sequential methotrexate-5-fluorouracil; and f, ramucirumab. Asterisk indicates the time of recurrence. AFP, alpha-fetoprotein; CEA, carcinoembryonic antigen.

three courses of irinotecan (15), and two courses of sequential methotrexate-5-fluorouracil chemotherapy (16). However, CT 
A

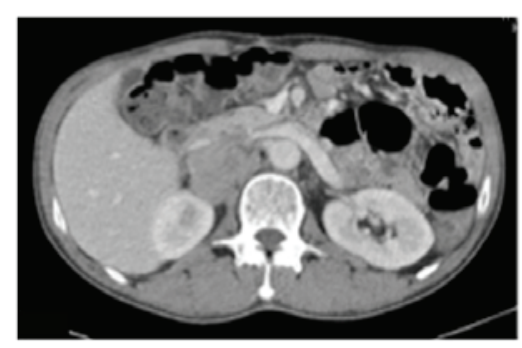

B

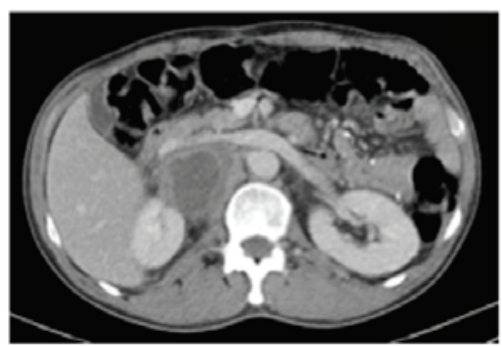

C

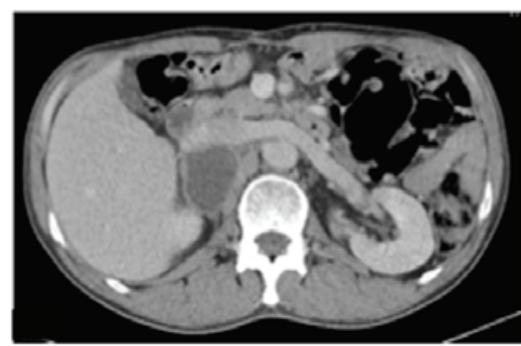

Figure 3. Contrast-enhanced computed tomography scans of the patient (A) prior to, and at (B) 3 and (C) 4 months after the initiation of ramucirumab monotherapy.

scan revealed that the metastatic para-aortic lymph node was gradually increasing in size, while the serum AFP level was increased to a maximum of 12,800 ng/ml (Figs. 2 and 3A).

The patient was then treated with ramucirumab monotherapy (7). Following six doses of ramucirumab, the patient presented with back pain and low-grade fever. Laboratory tests revealed an increased CRP level, at $11.23 \mathrm{mg} / \mathrm{dl}$ (normal range, $0-0.30 \mathrm{mg} / \mathrm{ml}$ ). Contrast-enhanced $\mathrm{CT}$ revealed a larger para-aortic lymph node with adjacent fat stranding, in addition to a significant decrease of enhancement in the middle of the lymph node, suggesting attenuation of vascularity (Fig. 3B). The patient was prescribed celecoxib for his back pain, and ramucirumab was discontinued for a month. Follow-up CT revealed a marked reduction of enhancement in the lymph node, suggesting an episode of central necrosis, while the serum AFP level decreased rapidly to $225 \mathrm{ng} / \mathrm{ml}$ (Figs. 2 and 3C). The patient proceeded with ramucirumab and has so far survived for 16 months after recurrence. Written informed consent was obtained from the patient for publication of this case report.

\section{Discussion}

AFPGC is considered a highly aggressive type of gastric cancer. The 5-year survival rate and median survival time are $42 \%$ and 29 months, respectively, even in patients who undergo curative surgical resection (17). Although molecular-targeted therapy has led to survival improvement of patients with various types of cancer $(7,18-20)$ there is no validated application of tailored therapy for this challenging subtype of gastric cancer. In the present case, the tumor cells were positive for VEGF. AFPGC is known to express high levels of VEGF or its isoform VEGF-C, and is considered to have increased neovascularization $(4,5,21)$. Since VEGF and VEGF-C serve as angiogenic factors in the tumor microenvironment (22), their inhibition may exhibit anti-angiogenic and anti-tumor activities on AFPGC. However, only a few cases of the efficacy of an anti-angiogenic multi-kinase inhibitor (namely sorafenib) in AFPGC patients have been reported thus far $(23,24)$. The clinical efficacy of ramucirumab, a monoclonal antibody for VEGF receptor-2, in AFPGC patients has not yet been reported. Sorafenib inhibits the signaling of the VEGF receptor and the platelet-derived growth factor receptor, but its specific targets are not fully understood yet. Sorafenib also inhibits the activity of the Raf/mitogen-activated protein kinase kinase/extracellular signal-regulated kinase and Janus kinase/Signal Transducers and Activator of Transcription signaling pathways $(25,26)$. Therefore, it is not clear that the efficacy of sorafenib in the previous AFPGC cases was due to its anti-angiogenic properties or its direct pro-apoptotic effects.

In the present case, the patient's metastatic lymph node exhibited marked central necrosis following six doses of ramucirumab monotherapy, which is similar to the effect of anti-angiogenic drugs on hepatocellular carcinoma (HCC), another AFP-producing cancer (27). Sorafenib has been reported to have a survival benefit in patients with advanced HCC (28). However, the response rate to sorafenib in that study was only $2 \%$, according to Response Evaluation Criteria in Solid Tumors (RECIST) (28). It has been demonstrated that the change in vascularization of the lesion is the crucial point for evaluating the response to anti-angiogenic drugs; thus, a modified RECIST was proposed (27). According to RECIST evaluation, the present patient's metastatic lymph node was defined as stable disease without any change in its greatest dimension, while according to the modified RECIST, the lesion was defined as partial response because the maximal dimension of continuous enhancement in the lesion was markedly reduced. In second-line treatment of advanced HCC, ramucirumab may have a survival benefit only for patients with high serum AFP levels (29).

Recent advances in the understanding of molecular pathogenesis have led to the development of molecular-targeted therapy in various types of cancer, including gastric cancer (7,18-20). In first-line treatment of HER2-positive advanced gastric cancer, the addition of trastuzumab to chemotherapy improves overall survival (7). Ramucirumab improves survival in the second-line setting of advanced gastric cancer; however, predictive biomarkers have not yet been clearly defined (30). In the present case report, the patient with chemotherapy-resistant recurrent AFPGC exhibited a significant response to ramucirumab monotherapy. In line with previous reports on AFPGC, the present patient's tumor cells were positive for VEGF. AFP production in gastric cancer may represent one of the predictors of ramucirumab sensitivity. The present case report demonstrates a potential new treatment option for AFPGC.

\section{References}

1. Kono K, Amemiya H, Sekikawa T, Iizuka H, Takahashi A, Fujii $\mathrm{H}$ and Matsumoto Y: Clinicopathologic features of gastric cancers producing alpha-fetoprotein. Dig Surg 19: 359-365 discussion 365, 2002. 
2. Inoue M, Sano T, Kuchiba A, Taniguchi H, Fukagawa T and Katai H: Long-term results of gastrectomy for alpha-fetoprotein-producing gastric cancer. Br J Surg 97: 1056-1061, 2010.

3. Li XD, Wu CP, Ji M, Wu J, Lu B, Shi HB and Jiang JT: Characteristic analysis of $\alpha$-fetoprotein-producing gastric carcinoma in China. World J Surg Oncol 11: 246, 2013.

4. Koide N, Nishio A, Igarashi J, Kajikawa S, Adachi W and Amano J: Alpha-fetoprotein-producing gastric cancer: Histochemical analysis of cell proliferation, apoptosis and angiogenesis. Am J Gastroenterol 94: 1658-1663, 1999.

5. Takahashi Y, Ohta T and Mai M: Angiogenesis of AFP producing gastric carcinoma: Correlation with frequent liver metastasis and its inhibition by anti-AFP antibody. Oncol Rep 11: 809-813, 2004.

6. Yuan DD, Zhu ZX, Zhang X and Liu J: Targeted therapy for gastric cancer: Current status and future directions (Review). Oncol Rep 35: 1245-1254, 2016.

7. Bang YJ, Van Cutsem E, Feyereislova A, Chung HC, Shen L, Sawaki A, Lordick F, Ohtsu A, Omuro Y, Satoh T, et al: Trastuzumab in combination with chemotherapy versus chemotherapy alone for treatment of HER2-positive advanced gastric or gastro-oesophageal junction cancer (ToGA): A phase 3, open-label, randomised controlled trial. Lancet 376: 687-697, 2010.

8. Fuchs CS, Tomasek J, Yong CJ, Dumitru F, Passalacqua R, Goswami C, Safran H, dos Santos LV, Aprile G, Ferry DR, et al: Ramucirumab monotherapy for previously treated advanced gastric or gastro-oesophageal junction adenocarcinoma (REGARD): An international, randomised, multicentre, placebo-controlled, phase 3 trial. Lancet 383: 31-39, 2014.

9. Wilke H, Muro K, Van Cutsem E, Oh SC, Bodoky G, Shimada Y, Hironaka S, Sugimoto N, Lipatov O, Kim TY, et al: Ramucirumab plus paclitaxel versus placebo plus paclitaxel in patients with previously treated advanced gastric or gastro-oesophageal junction adenocarcinoma (RAINBOW): A double-blind, randomised phase 3 trial. Lancet Oncol 15: 1224-1235, 2014.

10. Rüschoff J, Hanna W, Bilous M, Hofmann M, Osamura RY, Penault-Llorca F, van de Vijver M and Viale G: HER2 testing in gastric cancer: A practical approach. Mod Pathol 25: 637-650, 2012.

11. Okabe H, Hata H, Ueda S, Zaima M, Tokuka A, Yoshimura T, Ota S, Kinjo Y, Yoshimura K and Sakai Y; Kyoto University Surgical Oncology Group (KUSOG): A phase II study of neoadjuvant chemotherapy with S-1 and cisplatin for stage III gastric cancer: KUGC03. J Surg Oncol 113: 36-41, 2016.

12. Kinjo T, Taniguchi H, Kushima R, Sekine S, Oda I, Saka M, Gotoda T, Kinjo F, Fujita J and Shimoda T: Histologic and immunohistochemical analyses of $\alpha$-fetoprotein-producing cancer of the stomach. Am J Surg Pathol 36: 56-65, 2012.

13. http://link.springer.com/article/10.1245/s10434-010-0985-4

14. Sakuramoto $S$, Sasako $M$, Yamaguchi $T$, Kinoshita $T$, Fujii M, Nashimoto A, Furukawa H, Nakajima T, Ohashi Y, Imamura $\mathrm{H}$, et al: Adjuvant chemotherapy for gastric cancer with S-1, an oral fluoropyrimidine. N Engl J Med 357: 1810-1820, 2007.

15. Hironaka S, Ueda S, Yasui H, Nishina T, Tsuda M, Tsumura T, Sugimoto N, Shimodaira H, Tokunaga S, Moriwaki T, et al: Randomized, open-label, phase III study comparing irinotecan with paclitaxel in patients with advanced gastric cancer without severe peritoneal metastasis after failure of prior combination chemotherapy using fluoropyrimidine plus platinum: WJOG 4007 trial. J Clin Oncol 31: 4438-4444, 2013.

16. Hamaguchi T, Shirao K, Yamamichi N, Hyodo I, Koizumi W, Seki S, Imamura T, Honma H, Ohtsu A, Boku N, et al: A phase II study of sequential methotrexate and 5-fluorouracil chemotherapy in previously treated gastric cancer: A report from the gastrointestinal Oncology group of the Japan clinical oncology group, JCOG 9207 trial. Jpn J Clin Oncol 38: 432-437, 2008.

17. Adachi Y, Tsuchihashi J, Shiraishi N, Yasuda K, Etoh T and Kitano S: AFP-producing gastric carcinoma: Multivariate analysis of prognostic factors in 270 patients. Oncology 65: 95-101, 2003
18. Coiffier B, Thieblemont C, Van Den Neste E, Lepeu G, Plantier I, Castaigne S, Lefort S, Marit G, Macro M, Sebban C, et al: Long-term outcome of patients in the LNH-98.5 trial, the first randomized study comparing rituximab-CHOP to standard CHOP chemotherapy in DLBCL patients: A study by the Groupe d'Etudes des Lymphomes de l'Adulte. Blood 116: 2040-2045, 2010.

19. Mitsudomi T, Kosaka T, Endoh H, Horio Y, Hida T, Mori S, Hatooka S, Shinoda M, Takahashi T and Yatabe Y: Mutations of the epidermal growth factor receptor gene predict prolonged survival after gefitinib treatment in patients with non-small-cell lung cancer with postoperative recurrence. J Clin Oncol 23: 2513-2520, 2005

20. Van Cutsem E, Köhne CH, Láng I, Folprecht G, Nowacki MP, Cascinu S, Shchepotin I, Maurel J, Cunningham D, Tejpar S, et al: Cetuximab plus irinotecan, fluorouracil and leucovorin as first-line treatment for metastatic colorectal cancer: Updated analysis of overall survival according to tumor KRAS and BRAF mutation status. J Clin Oncol 29: 2011-2019, 2011.

21. Kamei S, Kono K, Amemiya H, Takahashi A, Sugai H, Ichihara F, Fujii $\mathrm{H}$ and Matsumoto Y: Evaluation of VEGF and VEGF-C expression in gastric cancer cells producing alpha-fetoprotein. J Gastroenterol 38: 540-547, 2003.

22. Ichikura T, Tomimatsu S, Ohkura E and Mochizuki H: Prognostic significance of the expression of vascular endothelial grow th factor (VEGF) and VEGF-C in gastric carcinoma. J Surg Oncol 78: 132-137, 2001.

23. Koneri K, Hirono Y, Fujimoto D, Sawai K, Morikawa M, Murakami M, Goi T, Iida A, Katayama K and Yamaguchi A: Five-year survival of alpha-fetoprotein-producing gastric cancer with synchronous liver metastasis: A case report. J Gastric Cancer 13: 58-64, 2013.

24. Fang YU, Wang L, Yang N, Gong X, Zhang YU and Qin S: Successful multimodal therapy for an $\alpha$-fetoprotein-producing gastric cancer patient with simultaneous liver metastases. Oncol Lett 10: 3021-3025, 2015.

25. Wilhelm SM, Adnane L, Newell P, Villanueva A, Llovet JM and Lynch M: Preclinical overview of sorafenib, a multikinase inhibitor that targets both Raf and VEGF and PDGF receptor tyrosine kinase signaling. Mol Cancer Ther 7: 3129-3140, 2008.

26. Blechacz BR, Smoot RL, Bronk SF, Werneburg NW, Sirica AE and Gores GJ: Sorafenib inhibits signal transducer and activator of transcription-3 signaling in cholangiocarcinoma cells by activating the phosphatase shatterproof 2. Hepatology 50: 1861-1870, 2009.

27. Edeline J, Boucher E, Rolland Y, Vauléon E, Pracht M, Perrin C, Le Roux C and Raoul JL: Comparison of tumor response by Response Evaluation Criteria in Solid Tumors (RECIST) and modified RECIST in patients treated with sorafenib for hepatocellular carcinoma. Cancer 118: 147-156, 2012.

28. Llovet JM, Ricci S, Mazzaferro V, Hilgard P, Gane E, Blanc JF, de Oliveira AC, Santoro A, Raoul JL, Forner A, et al: Sorafenib in advanced hepatocellular carcinoma. N Engl J Med 359: 378-390, 2008

29. Zhu AX, Park JO, Ryoo BY, Yen CJ, Poon R, Pastorelli D, Blanc JF, Chung HC, Baron AD, Pfiffer TE, et al: Ramucirumab versus placebo as second-line treatment in patients with advanced hepatocellular carcinoma following first-line therapy with sorafenib (REACH): A randomised, double-blind, multicentre, phase 3 trial. Lancet Oncol 16: 859-870, 2015.

30. Kim C, Mulder K and Spratlin J: How prognostic and predictive biomarkers are transforming our understanding and management of advanced gastric cancer. Oncologist 19: 1046-1055, 2014. 